\title{
Moving influenza vaccines forward
}

Expert Rev. Vaccines 8(4), 375-377 (2009)

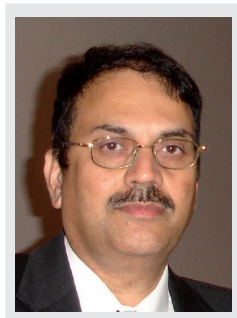

Suryaprakash Sambhara

Influenza Division, Centers for Disease Control and Prevention, Atlanta, Georgia, GA 30333, USA ssambhara@cdc.gov

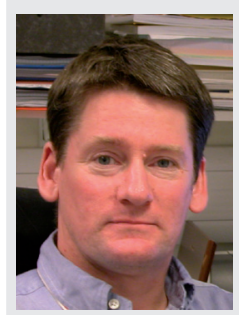

Iain Stephenson Author for correspondence Infectious Diseases Unit, University Hospitals Leicester, Leicester LE1 5WW, UK iain.stephenson@ uhl-tr.nhs.uk

\section{EXPERT
REVIEWS}

\section{"Pandemic influenza will cause huge morbidity, mortality and economic disruption when it next emerges."}

The WHO estimates that epidemic influenza affects approximately $5-15 \%$ of the global population annually, and is responsible for up to $3-5$ million cases of severe disease and 500,000 deaths per year [1]. The impact of influenza in developing countries is poorly described, suggesting that the real global burden of influenza-attributable disease is significantly underestimated. While influenza occurs among all age groups, the elderly and those with underlying chronic conditions are especially vulnerable and, in general, seasonal influenza vaccine campaigns focus on these high-risk groups. However, the importance of influenza in children is increasingly being recognized, and the expansion of influenza immunization programs to school-age children may afford herd immunity, reducing virus transmission to other groups, as well as providing individual protection. If shown to be effective, the introduction of widespread childhood immunization may become an important public-health tool for influenza control.

The world has experienced at least three documented influenza pandemics that resulted in severe infections and deaths of millions of people worldwide. The Spanish influenza H1N1 pandemic in 1918 was followed by the emergence of a new subtype, H2N2, which resulted in a pandemic beginning in 1957. The year 1968 marked the disappearance of the $\mathrm{H} 2 \mathrm{~N} 2$ virus and the emergence of the H3N2 subtype, causing a third influenza A pandemic. Even in countries where influenza surveillance is well established, the true impact remains difficult to quantify. Pandemic influenza will cause huge morbidity, mortality and economic disruption when it next emerges. It is estimated that a pandemic similar to 1968 occurring in 2004 would have killed 62 million people worldwide, with $96 \%$ of these deaths occurring in the developing world.

\section{“...widespread childhood} immunization may become an important public-health tool for influenza control."

The discovery of methods allowing large-scale influenza virus cultivation in embryonated chicken eggs, and reliable quantification of virus by hemagglutination, led to the production of the first inactivated vaccines and their evaluation in a series of placebo-controlled trials among US military recruits between 1942 and 1945 [2]. Vaccines were immunogenic and demonstrated a protective efficacy of $70-88 \%$ against natural infection with influenza $\mathrm{A}$ and $\mathrm{B}$. However, when a new influenza $\mathrm{A} / \mathrm{H} 1 \mathrm{~N} 1$ variant emerged in 1946-1947, the available vaccines provided little protection. The importance of close antigenic matching with the need to review vaccine strain selections was learned from this experience. Since their introduction into clinical practice, there have been improvements in methods of influenza vaccine purification, potency testing and standardization; however, the basic technology and principles of production have remained much the same since the 1940s.

Current inactivated influenza vaccines are trivalent, containing $15 \mu \mathrm{g}$ of hemagglutinin of two influenza $\mathrm{A}(\mathrm{H} 1 \mathrm{~N} 1$ and $\mathrm{H} 3 \mathrm{~N} 2$ ) subtypes and one influenza $B$ strain. Today, most commercially produced influenza vaccines are grown in eggs and are supplied as 'split subvirion' vaccines, produced from the disrupted, highly purified virus, or as a 'surface-antigen' vaccine containing predominantly purified hemagglutinin and neuraminidase, although 
vaccines are standardized based solely on hemagglutinin content. Depending on the vaccine purification process, vaccine preparations may also contain variable amounts of nucleoprotein and matrix proteins. Following vaccination, approximately $90 \%$ of healthy subjects reach serum hemagglutination-inhibition titers of greater than 1:40, a level generally associated with protection. Accurate assessment of vaccine effectiveness is challenging owing to varying case definitions of influenza, the use of different clinical end points and poor correlates of protection in immunogenicity studies. Protective efficacy of $70-95 \%$ against laboratory-confirmed influenza $\mathrm{A}$ can be achieved in young adults when there is a good match between vaccine and circulating strains. Although most influenza vaccine recommendations target high-risk and older subjects, current vaccines display lower effectiveness in these groups compared with younger adults. There is a clear and currently unmet need for vaccines with greater immunogenicity, especially for older adults.

\section{"Dose-sparing approaches are needed to enable} efficient use of limited antigen supplies, as global vaccine demand will outstrip production capacity."

The potential threat posed by highly pathogenic avian influenza $\mathrm{H} 5 \mathrm{~N} 1$ has prompted significant investments in pandemic-preparedness planning, a key component of which is the widespread availability of effective vaccines. The immune status of the population in a pandemic differs from that during the interpandemic period. A two-dose pandemic vaccine schedule is required to induce protective responses, posing logistic challenges to rapid production and delivery. Dose-sparing approaches are needed to enable efficient use of limited antigen supplies, as global vaccine demand will outstrip production capacity. Driven by pandemic preparedness and the requirement for enhanced immunogenicity, a great deal of research and development has taken place over the last decade, leading to improvements in vaccine production, antigen presentation, formulation with novel adjuvants delivery, which are now being implemented into clinical practice, making this supplement particularly topical and informative.

A significant and recent development in vaccine technology has been reverse genetics [3]. Since influenza possesses a segmented RNA genome, simultaneous infection of eggs with two different viruses may result in reassortment of segments to generate a new strain. Traditionally, the antigenically relevant strain is coinfected in eggs with a laboratory-adapted A/Puerto Rico/8/34 (H1N1) virus, leading to a reassortant containing the desired hemagglutinin and neuraminidase genes with the six remaining gene segments from PR/8/34 that confer high growth properties in eggs. This process requires large numbers of eggs and lacks flexibility if rapid response is needed. Furthermore, the use of highly pathogenic or nonhuman influenza viruses is limited by their lethality to eggs and biosecurity requirements. Reverse genetics permits the manipulation of viruses, including highly pathogenic subtypes, to rapidly generate seed strains for any potential pandemic virus by transfecting a cell with plasmids containing RNA segments. Gene mutations, insertions or deletions can be introduced to attenuate and optimize vaccine seed viruses. Vaccines prepared from reverse genetic-derived $\mathrm{A} / \mathrm{H} 5 \mathrm{~N} 1$ strains have been produced and clinically evaluated, and were first licenced by European and US regulators in 2007.

A further milestone is the licensure in 2007 by European regulators of the first seasonal influenza vaccine produced in a mammalian cell line (Madin-Canin-Darby-Kidney) rather than in eggs. Owing to the large number of embryonated eggs required for virus growth, seasonal vaccine production is a carefully planned process. Cell culture systems increase manufacturing consistency, reduce contamination risks and improve flexibility, particularly if vaccine production is required at short notice to meet unexpected demand. Other mammalian cell lines being used for influenza vaccine production include Vero and PerC-6 cells.

Various adjuvants have been used to enhance the immunogenicity of subvirion vaccines, but are typically associated with increased reactogenicity. Aluminium salts were added to seasonal influenza vaccines in the 1970 s, but did not produce benefits. Recent clinical studies of alum-adjuvanted pandemic vaccine candidates have failed to show consistent improvements in immunogenicity at lower vaccine doses. However, the addition of squalene-based oil-in-water emulsion adjuvants increases antibody responses and broadens immunity against nonvaccine strains. MF59-adjuvanted seasonal influenza vaccine was first licensed for use in 1997. AS03 was licensed for use in prepandemic vaccines in 2007 and others are under development. Enhanced immunogenicity of oil-in-water emulsion adjuvanted vaccines has been shown in elderly subjects, in those with chronic conditions and, more recently, in children. However, their most striking impact has been on pandemic vaccine formulation where recipients are immunologically naive to the antigen. Nonadjuvanted subunit $\mathrm{H} 5 \mathrm{~N} 1$ and $\mathrm{H} 9 \mathrm{~N} 2$ vaccines are poorly immunogenic in healthy adults, requiring two doses containing up to six-times the amount of antigen to induce acceptable responses compared with seasonal strains. The addition of MF59 or AS03 allows significant dose reduction while broadening and prolonging the protective antibody response to antigenically diverse strains. In addition, priming subjects with M59-adjuvanted $\mathrm{H} 5$ vaccine induces crossreactive B-cell memory responses that can be rapidly mobilized many years later by a single booster dose. These properties have led authorities to stockpile pandemic $\mathrm{H} 5$ vaccines and consider proactive prepandemic priming of key personnel to reduce logistic challenges in delivering rapid and effective pandemic vaccination schedules at the beginning of a pandemic [4].

\section{"...conventional vaccines based on hemagglutinin require regular updating to maintain a good match between the vaccine and circulating strain."}

Different methods of antigen presentation with or without adjuvant may be used for parenteral or mucosal vaccination. Liposomes are lipid membrane particles that serve as delivery systems for antigens. Virosomes are created by inserting influenza hemagglutinin and neuraminidase into a liposome bilayer that presents antigen more similar to an intact virion, thus enhancing 
cellular responses. Proteosomes are nanoparticles formed from meningococcal outer membrane proteins that induce potent antigen-specific mucosal and systemic responses when combined with influenza hemagglutinin.

Alternatives to egg-derived hemagglutinin include the use of technologies to produce antigens in modern expression systems, such as recombinant engineered baculovirus in insect cells or antigen expression in plant cells, which should make manufacturing more efficient and robust. Potential difficulties with recombinant vaccines include the use of uncleaved hemagglutinin and differences in glycosylation that may affect immunogenicity. Nonetheless, dose-related antibody responses to baculovirusexpressed trivalent seasonal vaccines appear to be similar to those induced by conventional vaccines. Baculovirus $\mathrm{H} 5$ and $\mathrm{H} 7$ hemagglutinin vaccines protect against lethal challenge in chickens even when challenge viruses are antigenically distinct. However, when clinically assessed, a recombinant baculovirus $\mathrm{H} 5$ vaccine was suboptimal, suggesting that improvements in immunogenicity or delivery are needed. Virus vectors,such as adenovirus and vaccinia virus, can be genetically modified to express and deliver selected influenza proteins by parenteral or mucosal routes. Engineered virus vectors expressing influenza antigens offer broader immunity by induction of influenza-specific cellular responses, as well as systemic antibody. Since the antigenic characteristics of influenza surface proteins change over time, conventional vaccines based on hemagglutinin require regular updating to maintain a good match between the vaccine and circulating strain. However, internal viral antigens are highly conserved, leading to the idea that a 'universal' influenza vaccine against all subtypes based on internal proteins such as M2 may be possible to confront pandemic threats, perhaps as an additive to the existing vaccine.

In summary, commercial influenza vaccine production is based on inactivated egg-grown reassortant PR8 virus using basic manufacturing processes developed over 50 years ago. Substantial research over the last decade has led to the regulatory approval and clinical introduction of reverse-genetic engineered vaccines, cell-culture grown vaccines, alternative delivery routes and potent oil-in-water emulsion adjuvants. Alternative methods of antigen presentation such as virus-like particles and virosomes, with or without adjuvant, could further enhance immunogenicity. It is only a matter of time before the potential of recombinant antigens using different substrates, expression systems and modes of delivery (e.g., virus vectors) is realized with appropriate formulations and delivery technologies in safety and immunogenicity trials. We hope that the collection of expert reflections in this supplement will both be enjoyed by and educate the reader.

\section{Financial \& competing interests disclosure}

The findings and conclusions in this report are those of the authors and do not necessarily represent the views of the funding agency or the CDC. The authors have no other relevant affiliations or financial involvement with any organization or entity with a financial interest in or financial conflict with the subject matter or materials discussed in the manuscript apart from those disclosed.

No writing assistance was utilized in the production of this manuscript.

\section{References}

1 WHO Influenza Fact sheet 211. World Health Organisation, Geneva, Switzerland (2003).

2 Francis T. Vaccination against influenza. Bull. World Health Organ. 8, 725-741 (1953).
3 Webby RJ, Perez DR, Coleman JS et al. Responsiveness to pandemic alert: use of reverse genetics for rapid development of influenza vaccines. Lancet 363, 1099-1103 (2004).
4 Jennings LC, Monto AS, Chan PK, Szucs TD, Nicholson KG. Stockpiling prepandemic influenza vaccines: a new cornerstone of pandemic plans. Lancet Infect. Dis. 8(10), 650-658 (2008). 